\section{Physiotherapy Treatment of Surgical Chest Conditions}

\author{
By MARGARET R. WHITE, M.C.S.P., \\ Lecturer, Department of Physiotherapy, \\ University of Cape Town.
}

From the point of view of the treatment of Surgical Chest conditions by Physiotherapy, this article can only hope to state broad outlines of treatment which will have to be modified and varied according to the type of case-and the personality of the patient-to be treated.

An attempt has been made to classify the types of case suitable for surgery under very wide headings. A common condition and its normal treatment and progression has then been chosen with some comment on modification in treatment according to different types of case. Concentration has been placed on basic techniques which should thus act as a guide for the treatment of other more complicated chest conditions.

For the purpose of this artlcle surgical chest conditions can be divided into 3 broad sections:

$$
\begin{aligned}
& \text { 1. Non T.B. Cases } \\
& \text { 2. T.B. Cases } \\
& \text { 3. Heart Cases }
\end{aligned}
$$

Before starting to discuss treatments it must always be remembered that the Physiotherapist is only one member of a team, and the more closely the members of that team work together, the more effective are the results. Perhaps in Thoracic surgery more than in any other branch of surgery the patient's condition varies from hour to hour, and for fully effective treatment it is essential that the Physiotherapist is kept informed of any changes throughout the day.

1. Excluding heart surgery perhaps the greatest majority of surgical chest cases to-day falls into the Bronchiectatic and Carcinoma groups, and in this article the pre and post operative treatment of one of the most usual operations, Lobectomy, where a Thoracotomy is performed, will be discussed.

To prepare the patient for operation a minumum of three pre operative sessions is required, although one week's preparation is more desirable.

Certain conditions will, of course, require longer pre operative treatment. For example, Bronchiectasis where, in a bad case, some weeks of carefully supervised Postural Drainage will be necessary to clear the chest before operation, thus helping to prevent post operative collapse caused by an overflow of secretions into neighbouring bronchi.

Not only does the patient need to be instructed in breathing i control, correct coughing, etc., but it is a great help to him to get to know the ward routine and the members of staff who will be caring for him after operation. He will thus learn to have confidence in them and know how they can help him as well as learning how to help himself.

A simple explanation of where his scar will be, why he has to cough and how his chest will be supported when coughing, will all be found helpful and re-assuring to the patient.

Pre-operative Treatment can be divided into 5 main groups:

To teach (1) correct breathing control both Diaphragmatic and localised and correct coughing

(2) maintenance of posture

(3) localised arm movements

(4) foot and leg exercises with emphasis on importance of moving about in bed

(5) and supervise the correct Postural Drainage positions in cases where this is necessary.

\section{(1) Correct Breathing Control.}

First correct Expiratory Breathing will have to be taught in order to help to empty the chest of Post operative secretions, followed by careful Inspiratory localised breathing, bearing in mind the type of operation to be performed.
For Pneumonectomies - Diaphragmatic Control and careful localised breathing to the base of the lung on the sound side are most important.

For Lobectomies, as well as diaphragmatic control, all areas on the operation side will have to be treated so that the remaining lung tissue will re-expand fully, as soon as possible, to fill the whole of the space on the operation side.

Teach how to cough. Short sharp expirations with contraction of the abdominal muscles should first be taught and practised. It is then explained how this is practically a cough. Next, coughing is practised making sure that the cough comes right from the base of the chest.

\section{Maintenance of Posture}

The type of deformity likely to arise after thoracic operation varies according to the operation performed.

Following Thoracotomy (either for lobectomy or for pneumonectomy) the patient tends to lift the hip on the operation side while putting all the weight on the hip on the sound side. The shoulder on the operation side is dropped while the arm is held tightly in to the side, thus producing a C curve away from the side of the operation. The patient should be taught (a) alteration of weight-bearing on each hip; (b) with the elbows bent down-pressing of elbow on the sound side; (c) back raising vertebra by vertebra.

A mirror that can be placed at the end of the bed is invaluable in teaching, maintaining and correcting posture.

\section{Localised Arm Movements}

Small localised arm movements should be taught preoperatively as the retractors of scapulae are often cut through at operation, and the patient is generally frightened and unwilling to move the arm immediately post-operatively.

Teach- (a) Retraction of scapulae

(b) Abduction of arm and forward flexion (with elbow bent and hand resting on shoulder to begin with)

(c) Elevation of arm with the assistance of the other hand to begin with.

\section{Foot and Leg Exercises}

The importance of these exercises need only be mentioned in passing, they are routine post operative treatment. The patient after Thoracotomy is of ten so afraid to move at all that special instruction in the importance of moving about in bed and re-assurance that no harm will result, and that it can only do good, saves a certain amount of post-operative tension.

\section{Correct Postural Drainage}

If postural drainage is to be efficient, much emphasis must be placed on the importance of correct breathing during the postural drainage sessions. One might almost say that one half of the success of Postural drainage lies in seeing that the patient concentrates on Expiratory diaphragmatic breathing while posturing, thus greatly assisting the expectoration of sputum.

The other half-lies in Correct Positioning and Percussion with localised breathing. It is outside the scope of this article to describe in detail each Postural Drainage position, but the Red $X$ have published a leaflet illustrating these positions, and Miss W. T. Thacker, M.C.S.P., has published an excellent booklet on the subject. The patient may often need encouragement and explanation on the importance of coughing and expectoration. They must have no fine feelings on the matter of spitting, it is what is required. When the bronchial tree is efficiently drained of toxic secretions the patient's whole demeanour, mental and physical, show a dramatic improvement.

These pre-operative bronchiectatic patients should take part in a general limbering up class which includes breathing exercises and postural training, and should therefore be well prepared for operation.

\section{Post Operative Treatment}

As the type of case under consideration is that of Lobectomy it might be well to compare the post operative treatment and progress of a case of Lobectomy for Bronchietasis with one for Carcinoma. 
In Bronchietasis the patient is usually young with a good prognosis. He will be expected to progress quickly to a full active life. Care should be taken to explain to him that he is no longer an invalid and that he can now go out and take part in any type of active exercise he fancies. So often these patients have lived a sheltered inactive existence due to the restrictions and embarrassments that the constant cough and sputum have forced upon them. It is difficult for them to realise that they can-and should-lead a healthy and moderately energetic life when they recover from the immediate post operative effects.

Their progress in hospital should be swift, they should be able to attend an exercise class 7 to 10 days after operation and be discharged any time after 14 days.

In Carcinoma the patient is usually older and in poor general health. It is known that he has not a long expectancy of life and that - at least generally - this has been a palliative operation. Progress will, therefore, be somewhat slower and he will not be expected to progress to full activity so quickly, particularly in the older age group. Though it is, of course, desirable to have him discharged as soon as possible, so that he may return to his home surroundings at the first opportunity. These patients tend to be breathless and, the pneumonectomies particularly, should always be taught walking with controlled breathing, and also stair climbing with controlled breathing as a routing before they leave hospital.

From the pre operative instructions it follows that our Post Operative Aims are:

1. To clear the chest of secretions, and obtain full lung expansion and function as soon as possible

To 2. maintain correct posture

3. maintain full arm function

4. encourage foot and leg exercises for circulatory purposes

5. return the patient to as full normal activity as possible-as soon as possible.

To obtain these results on the evening of operation the patient should be visited, if conscious, and correct breathing control started and the patient encouraged to try to cough. It is the first 2 or 3 days that are most vital in preventing post operative collapse of lung, and it is essential to see that the patient uses his diaphragm, thus moving his chest and helping early expectoration of sputum.

It is at this stage that the co-operation of the nursing staff is so essential. They too must realise that the patient must be encouraged to cough and spit at regular intervals, and they too must know how to support his chest, thus giving him confidence to cough deeply. The nursing staff must never be given the idea that "coughing the patient" is purely the Physiotherapist's job.

\section{Post Operative Postural Drainage}

In a case of bronchietasis, this should be started the morning after operation and carried out morning and afternoon until the $X$-ray shows that the lungs are clear and fully re-expanded. If there is still an area-of bronchietasis on the opposite side, the patient will have to continue to do postural drainage for the affected area.

The patient will probably have 2 tubes, an apical and a lower tube, in his pleural cavity for the first 24 to 28 hours, or until all drainage of the pleural space is finished. Once the tubes are removed the patient can get up and activity should be progressed as rapidly as possible.

The patient should be visited at least 4 times for the first two or three post operative days, the first and third visits being for postural drainage (which includes supervision of correct breathing) and correction of posture following drainage; the second and fourth visits principally to supervise correct breathing and coughing with support at the site of operation.

\section{Support While Coughing}

This support can be given with the patient leaning back against the pillow when the operator's hands are placed above and below the scar (the exact position varies according to what the patient finds most comfortable). When the

\section{This hygienic} Physiotherapist Uniform has so many good points!

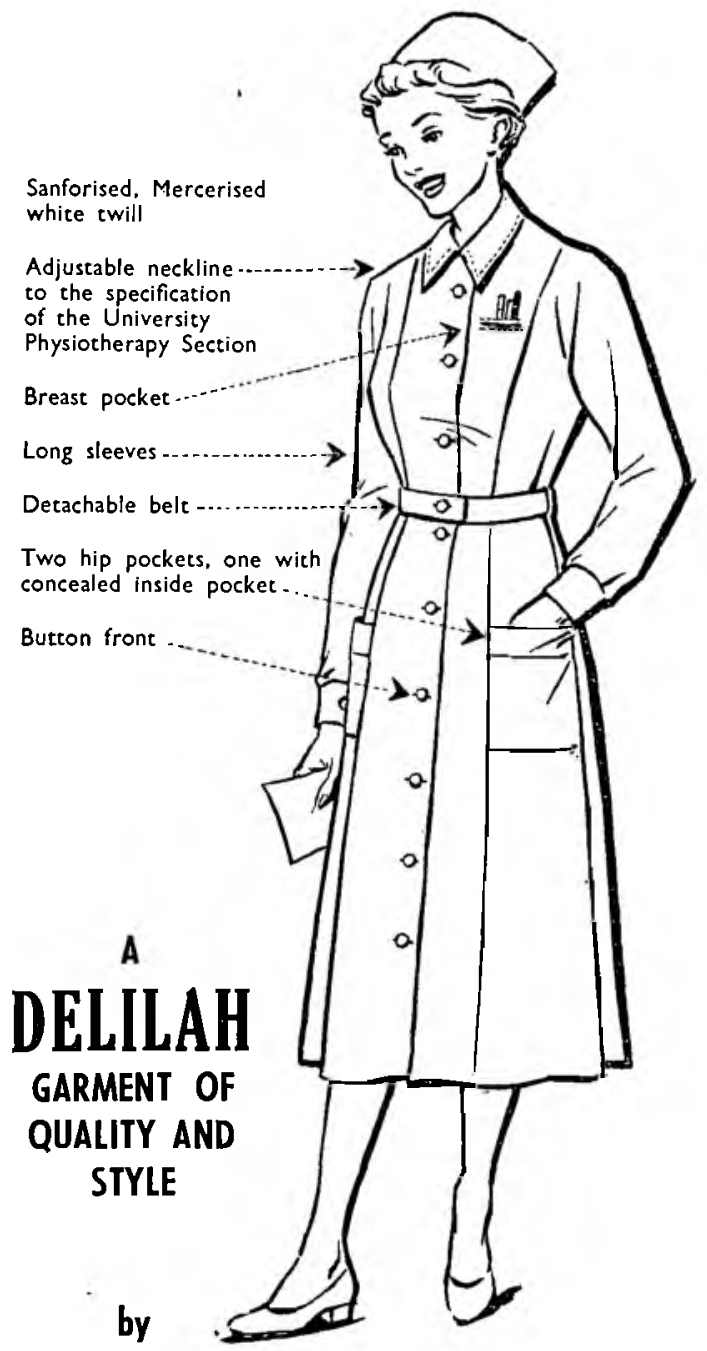

THE HOUSE OF ENSIGN

Manufactured by

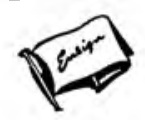

The AFRICAN CLOTHING FACTORY (Eusign) Ltd.

\section{P.O. BOX 1098 CAPE TOWN}

and obtainable from good clothing stores everywhere 
patient is sitting away from the pillows to cough, support an me more adequately from the sound side, with the operator's arm under the sound arm of the patient, me patient's chest supporting the chest wall below the scar, while the other arm goes across the patient's back and supports the chest above the scar. It is most important that the patient should have this chest support whenever possible for the first few post operative days. He is thus assisted and given confidence to cough more deeply and adequately.

Localised breathing to all areas of the operation side are also concentrated upon, thus obtaining mobility of the chest and full re-expansion of the remaining lung tissue as soon as possible. Posture, arm and leg exercises are checked.

Once the lung is well re-expanded (usually about the third or fourth day) the number of treatments can be decreased to 2 a day, and once the patient is attending class 1 other treatment should be sufficient.

In cases of carcinoma, post operative postural drainage may not always be required, though when in doubt it is safer to give postural drainage than to omit it, provided the surgeon has been consulted.

With regard to other Non T.B. conditions, the method of treatment is very similar, except that generally speaking there is less likelihood of post operative collapse as the chest secretions are less.

2. T.B. Cases

In T.B. Cases the most common surgical procedures are:

(a) Decortication following Empyema-where maximum emphasis is placed on intensive localised breathing exercises, to encourage lung expansion out to the chest wall at the earliest possible moment;

(b) Thoracoplasty-performed to collapse the affected area of lung, usually in the upper lobe. Here the main object is to prevent deformity which can arise so quickly due to muscular and skeletal inbalance. Careful pre-operative instruction is essential, particularly in correcting body lean, and neck and head deviation from midline. Care must be taken to see that the function of the base of the lung on the operation side is maintained.

(c) Lobectomy and Pneumonectomy-where the same rules apply as in the case already discussed.

3. Heart Cases-can be sub-divided into:

(a) Simple Operations - where the patient is up on the third to the fifth day; i.e. uncomplicated Mitral Valvotomy Patent Ductus Arteriosus.

(b) More complex operations - where the patient is not up until the 10th to the 14th day.

i.e. (1) All aortic Conditions-Aortic Stenosis Aortic Incompetence Co-arctation of Aorta

(2) All operations where a ventricle is opened Fallot's Tetralogy, Atrial Septal Defect, Pulmonary Valve Stenosis, Ventral Septal Defect.

Heart conditions do not generally require as much attention as the conditions previously discussed, as on the whole, there are fewer chest secretions. The chief points to remember are to regain and maintain mobility of the thorax and to clear the chest of secretions as early as possible. Acknowledgements:

"Physiotherapy for Chest Diseases"-Miss J. M. W. Reed,

"Postural Drainage"_-Miss W. Thacker, M.C.S.P. M.C.S.P.

Lecture on Surgical Heart Conditions-Miss S. Evans, M.C.S.P. (delivered at Paris Congress).

My thanks are due to Miss M. H. S. Roper, M.C.S.P., for reading these notes and for helpful suggestions.

\section{THE ENTRAX tRACTION UNIT}

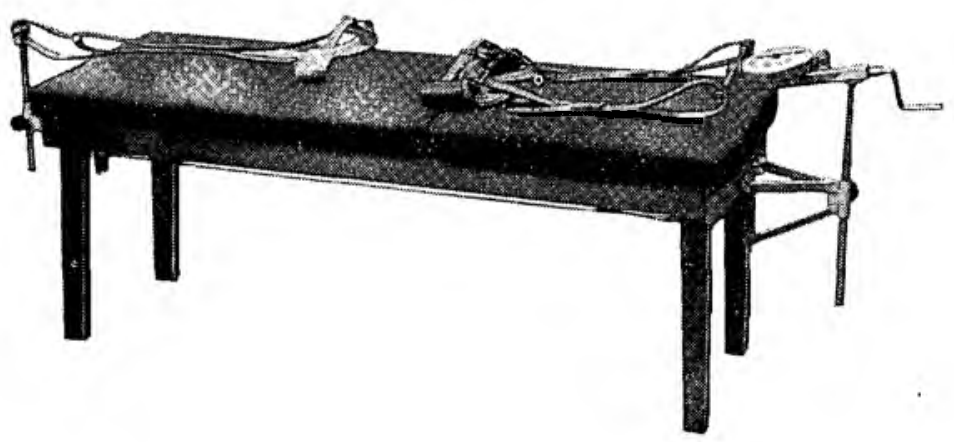

COMPREHENSIVE ADJUSTMENT

The ENTRAX allows for the alternatives you will need.

Mobility and direction of pull from either thorax or pelvis.

Changed in 2 minutes.

The thoracic harness enables either a posterior pull, anterior pull, or both combined. Up to 200 lbs. Lateral adjustability of the points of pull to suit various builds, with both thoracic and pelvic harness.

Cervical traction.

A fully adjustable halter is included.

The fittings fold quickly under ends of plinth without detaching, affording a clear couch top immediately. 